\title{
Relevance of system size to the steady-state properties of tapped granular systems
}

\author{
Paula A. Gago, ${ }^{1,2, *}$ Diego Maza, ${ }^{3}$ and Luis A. Pugnaloni ${ }^{1,2, \dagger}$ \\ ${ }^{1}$ Departamento de Ingeniería Mecánica, Facultad Regional La Plata, Universidad Tecnológica Nacional, \\ Av. 60 Esq. 124, 1900 La Plata, Argentina \\ ${ }^{2}$ Consejo Nacional de Investigaciones Científicas y Técnicas, Argentina \\ ${ }^{3}$ Departamento de Física y Matemática Aplicada, Facultad de Ciencias, Universidad de Navarra, Irunlarrea S/N, 31080 Pamplona, Spain
}

(Received 23 January 2015; published 30 March 2015)

\begin{abstract}
We investigate the steady-state packing fraction $\phi$ and force moment tensor $\Sigma$ of quasi-two-dimensional granular columns subjected to tapping. Systems of different height $h$ and width $L$ are considered. We find that $\phi$ and $\Sigma$, which describe the macroscopic state of the system, are insensitive to $L$ for $L>50 d$ (with $d$ the grain diameter). However, results for granular columns of different heights cannot be conciliated. This suggests that comparison between results of different laboratories on this type of experiments can be done only for systems of same height. We show that a parameter $\varepsilon=1+(A \omega)^{2} /(2 g h)$, with $A$ and $\omega$ the amplitude and frequency of the tap and $g$ the acceleration of gravity, can be defined to characterize the tap intensity. This parameter is based on the effective flight of the granular bed, which takes into account the $h$ dependency. When $\phi$ is plotted as a function of $\varepsilon$, the data collapses for systems of different $h$. However, this parameter alone is unable to determine the steady state to be reached since different $\Sigma$ can be observed for a given $\varepsilon$ if different column heights are considered.
\end{abstract}

DOI: 10.1103/PhysRevE.91.032207

PACS number(s): 45.70.Cc, 05.20.Gg

\section{INTRODUCTION}

Granular materials, defined as multiparticle systems that interact via dissipative contact forces, pose significant challenges to the physics community. In some cases, even the correct variables that fully describe the states and/or processes are being debated. Of particular importance to the advancement of the description, explanation, and prediction of phenomena in physics is the knowledge of the necessary conditions to be able to reproduce results independently. This gives information on the relevant variables involved. In granular matter, there are still various examples of phenomena that cannot be reproduced at a quantitative level; therefore, results from different research groups are usually compared only in qualitative terms. One such phenomena is the steady state of tapped granular beds.

Stationary states of static granular packs subjected to pulsed excitations have been considered by a number of workers [1-13]. In recent years, the granular community has been focused on finding the correct variables to describe the macroscopic stationary states of static granular packs. There is now partial agreement that the volume $V$ (or, equivalently, the packing fraction $\phi$ ) and the force moment tensor $\Sigma$ are necessary to fully describe these states $[7,8,14-16]$. However, due to the difficulties of measuring $\Sigma$ in the laboratory, most studies focus only on the volume of the system. Although there exists general agreement on the qualitative behavior of $V$ (or $\phi$ ), quantitative comparisons between results obtained by different groups are still elusive. We hypothesize that the main cause for quantitative disagreement in the mean volume is related to the effect of the size of the samples used in each particular setup. Specifically, we will show that, although the width of the system is irrelevant for samples wide enough, the height of the granular column is a critical parameter in the problem and should be included in the description. Notice

\footnotetext{
*paulaalejandrayo@gmail.com
}

†luis.pugnaloni@frlp.utn.edu.ar that we consider systems under gravity, where a preferential direction is naturally present.

In this work, we consider the effect that the size of a granular sample confined in a quasi-two-dimensional (2D) cell has on the steady-state properties when subjected to tapping. We study $\phi$ and $\Sigma$ as a function of tap intensity for different system widths and heights using a discrete element method (DEM) simulation.

The rest of the paper is structured as follows. In Sec. II we describe the numerical model. In Sec. III we state the tapping protocol used, the system sizes investigated, and the techniques used to compare results from different systems. In Sec. IV we show the results for $\phi, \Sigma$ and their corresponding fluctuations in the steady state. In Sec. V we introduce a parameter that enables a better characterization of the effect of the external perturbation. Finally, in Sec. VI, we summarize the main results and draw the conclusions.

\section{NUMERICAL MODEL}

We use LAMMPS for our DEM simulations [17]. We simulated a quasi-2D cell containing a number $N$ of spherical particles of diameter $d$ and mass $m$ in a gravity field of acceleration $g$. We choose a quasi-2D cell ( $1.1 d$ in thickness) to obtain a realistic representation of usual experimental devices, where direct imaging can be used $[7,18]$. We use a model for soft dissipative spheres as described in Refs. [19,20]. The contact interactions involve a normal force and a tangential force. The normal component is given by a restoring force, proportional to the overlap of the interacting spheres, and a dissipative term, proportional to the normal component of the relative velocity. The tangential term implements an elastic shear force and a damping force. The shear force has a "history" effect that takes into account the cumulated tangential displacement between the particles while they remain in contact. Moreover, whenever the tangential force exceeds $\mu F_{\text {normal }}$, where $\mu$ is the friction coefficient, the dynamic friction force is used. In this work we use the same interaction parameters as in Ref. [6]: 
friction coefficient $\mu=0.5$, normal elastic constant $k_{n}=$ $10^{5}(\mathrm{mg} / \mathrm{d})$, normal dissipative constant $\gamma_{n}=150(\mathrm{~m} \sqrt{\mathrm{g} / \mathrm{d}})$, tangential elastic constant $k_{s}=\frac{2}{7} k_{n}$, and tangential dissipative constant $\gamma_{s}=100(m \sqrt{g / d})$. The integration time step is set to $\delta=10^{-4} \sqrt{d / g}$. The wall-particle interaction is defined with the same parameters as the particle-particle force.

\section{PROBLEM DEFINITIONS}

\section{A. Annealing protocol by tapping}

We apply a protocol of annealing by tapping similar to the one proposed in Ref. [21]. However, we only run a decreasing ramp of tap intensities. Starting from the highest intensity investigated, we decrease its value in discrete steps every 200 taps. Tapping is simulated by imposing an external vertical motion to the cell (walls and base) of the form of a full cycle of a sine wave: $A \sin (\omega t)$. We fix $\omega=2 \pi \times 33 \mathrm{~Hz}$ and use the tap amplitude $A$ as the control parameter. We consider the properties of the static (mechanically equilibrated) configurations obtained after each tap. Mechanical equilibrium is deemed achieved if the kinetic energy of each particle has fallen (in average) below $10^{-6} \mathrm{mgd}$.

We focus on the regime of high tap intensities where the packing fraction as a function of the reduced acceleration $\Gamma=$ $A \omega^{2} / g$ becomes nonmonotonic [6-8]. It has been recently shown that at relative low $\Gamma$ the series of configurations in the steady state is not ergodic $[22,23]$. We stop decreasing $\Gamma$ before the system reaches the regime where ergodicity of the stationary states is lost, according to the criteria proposed in Ref. [22].

\section{B. System sizes}

We consider different cell widths $L$ filled with different number $N$ of particles. We define the nominal height $h$ of the granular column as the integer part of $N / L$. The actual height for a given static configuration of the grains depends on the preparation protocol. In all the cases we have chosen $L$ to be noncommensurate with $d$ to prevent crystallization to some extent. In the following, we will use the pair $L-h$ to identify each system size. Figure 1 summarizes the sets of $L-h$ studied. We can distinguish sets with the same $h$ (varying $L$ ), sets with the same $L$ (varying $h$ ), and sets with the same aspect ratio (L1-h1, L2-h2, and L3-h3).

\section{Data analysis}

In the following, we shall call a "realization" to any full annealing process, i.e., 200 taps for each value of $\Gamma$ in a complete decreasing ramp. In all cases we disregard the initial 100 configurations obtained for each $\Gamma$ and only use the last 100 configurations to make averages. For the relatively high tap intensities we use in this work, this is sufficient to ensure that a stationary state has been reached for any given $\Gamma$. We repeat typically 20 independent realizations for each system size considered.

To measure the packing fraction $\phi$ we use the Voronoi tessellation of the plane using the coordinates of the centers of the particles projected on the $x-z$ plane, where $x$ is the direction along the cell width and $z$ is the vertical coordinate. Then we associate to each particle a $\phi_{\text {local }}$ by dividing the particle area (projected on the $x-z$ plane also) by the corresponding Voronoi

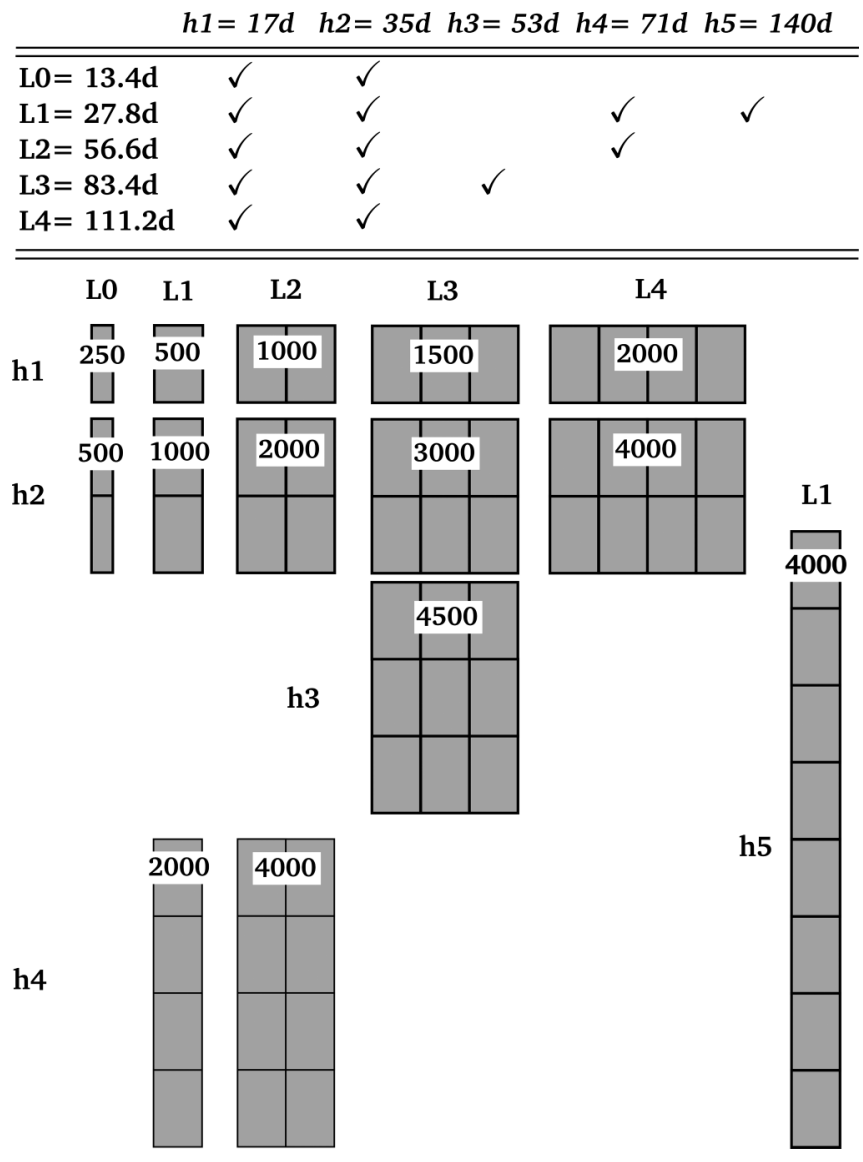

FIG. 1. Width $L$ of the cells and nominal height $h=\operatorname{int}(N / L)$ of the granular columns studied. The bottom image shows a schematic representations of the system sizes. The number on each cell corresponds to the number $N$ of particles used in each case. The numbers next to the $L$ and $h$ symbols will be used to identify any given sample by stating the pair $L-h$ (e.g., $L 2-h 4)$ in the rest of the paper.

cell area. By taking the average over all the particles in the region of interest (see next section for a discussion on the region where properties are measured), we obtain the global $\phi_{\text {conf }}$ corresponding to each static configuration. We average this quantity over the 100 configurations saved for each tap intensity to obtain the $\phi_{\text {realiz }}$ corresponding to the stationary states of the realization for each $\Gamma$. We calculate the packing fraction fluctuations $\Delta \phi_{\text {realiz }}$ as the standard deviation over the 100 configurations. Finally, we carry out a last average over the $\phi_{\text {realiz }}$ and the $\Delta \phi_{\text {realiz }}$ obtained in the 20 independent realizations to obtain $\phi$ and $\Delta \phi$ and their corresponding errors (using the standard deviation over the 20 realizations).

We also obtain the force moment tensor $\Sigma^{\alpha \beta}=\sum_{c} r_{c}^{\alpha} f_{c}^{\beta}$ of each particle. Here $c$ runs over all the contacts of the particle, $\boldsymbol{r}_{c}$ is the vector from the center of the grain to the contact $c$ and $\boldsymbol{f}_{c}$ is the corresponding contact force. We apply the same averaging protocol as the one used for $\phi$ to obtain the $\Sigma_{\text {realiz }}^{\alpha \beta}$ and its fluctuations $\Delta \Sigma_{\text {realiz }}^{\alpha \beta}$ corresponding to the realization and then use the 20 realizations to estimate the errors.

For all the tap intensities and system sizes studied, and the necessary statistics, in total $\approx 5 \times 10^{5}$ taps were simulated. 


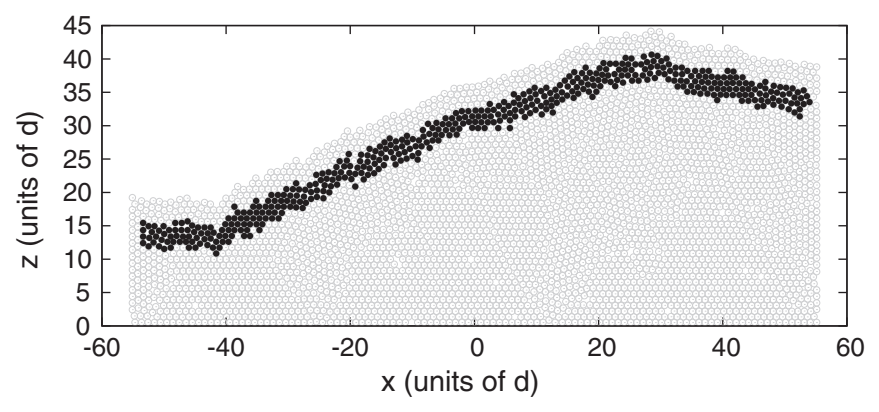

FIG. 2. Snapshot of a static configuration for a system with $L 4-h 2$. As an example, the particles in a typical layer used to measure $\phi$ and $\Sigma$ are highlighted. In this case the layer is $5 d$ in thickness and it is located at $5 d$ below the free surface.

Each tap requires between $10^{5}$ and $10^{7}$ simulation time steps, depending on the tap intensity and the system size.

\section{Analysis regions}

In order to compare results between systems of different sizes we needed to chose suitable regions of the system where to measure $\phi$ and $\Sigma$. For all the systems, we consider a thin layer of the sample that follow the shape of the free surface (see Fig. 2) at a given depth, including in the analysis only particles whose centers fall in this region. Since $\phi$ and $\Sigma$ are inhomogeneous in the vertical direction due to gravity, using a layer warrants that we focus on a region where a few macroscopic variables can describe the macrostate without the presence of significant spatial variations of the mean $\phi$ and $\Sigma$ in the region. Additionally, the free surface of the column of grains is not horizontal in general, especially for wide cells as described by others [4,9,24,25] (see also Fig. 2). We have observed that the simple use of plane horizontal layers to measure $\phi$, and more importantly $\Sigma$, leads to inconsistent results whenever modulations of the free surface are present. This occurs because particles with the same $z$ coordinate may support, effectively, different weights above them. Analyzing layers that follow the free surface profile has enabled us to compare results between different systems and different realizations consistently. We expect, however, that this detail may become of little relevance if very deep layers (buried at a depth a few times the width of the system) are studied.

We have observed that for layers of thicknesses above $3 d$ all the properties measured converge to well-defined values. Exemption to this rule are the fluctuations $\Delta \phi$ and $\Delta \Sigma$, which require thicker layers to converge (see Sec. IV C). The dependence of the macroscopic properties with depth will not be investigated here. We will limit our study to layers relatively close to the surface, where pressure as a function of depth is still linear. Unless otherwise stated, measurements are carried out over a layer of thickness $3 d$ at a depth $10 d$ below the free surface. In addition, we exclude particles with centers less than $2 d$ away from the lateral walls to minimize boundary effects. With this choice, the layer of analysis will contain different number of particles if systems of different $L$ are considered. When comparing systems of different $h$, we expect that layers at the same depth have similar properties if the pack is subjected to similar perturbations. We will see that this is not the case even if the effect of the column height on the effective perturbation suffered by the layer of interest is taken into account.

\section{RESULTS}

We report the results using the set of reduced units: $m=1$, $g=1$, and $d=1$.

\section{A. Packing fraction}

In Fig. 3(a) we show $\phi$ versus $\Gamma$ for all systems summarized in Fig. 1. This function presents a nonmonotonic behavior for all the systems studied, in agreement with previous works [6-8,26]. Previous studies were carried out with rather small systems (comparable with the system $L 0-h 1$ in the present work). Figure 3(a) implies that nonmonotonicity in the $\phi-\Gamma$ curve cannot be attributed to a finite-size effect.

It is important to notice that the amplitude $\Gamma_{\min }$ at which the system reaches the minimum packing fraction $\phi_{\min }$ clearly differs for different system sizes. Figures 3(b) and 3(c) show $\phi$ versus $\Gamma$ for two different system heights ( $h 2$ and $h 4$, respectively) and various cell widths. We can see that the curves collapse for systems with the same height, to a certain extent, and this collapse is better for low values of $\Gamma$. Figure $3(d)$ displays the results only for systems of width $L 1$ and different $h$. In this case the curves shift towards larger $\Gamma$ for taller samples.

The results on Fig. 3 indicate that the steady-state packing fraction is unique for a given tap intensity only if the columns have the same nominal height. Varying the width of the sample does not influence the steady state significantly. However, the height of the sample is determinant. This can be understood if one considers that the effective perturbation felt by the entire system depends on the column height. The same tap applied
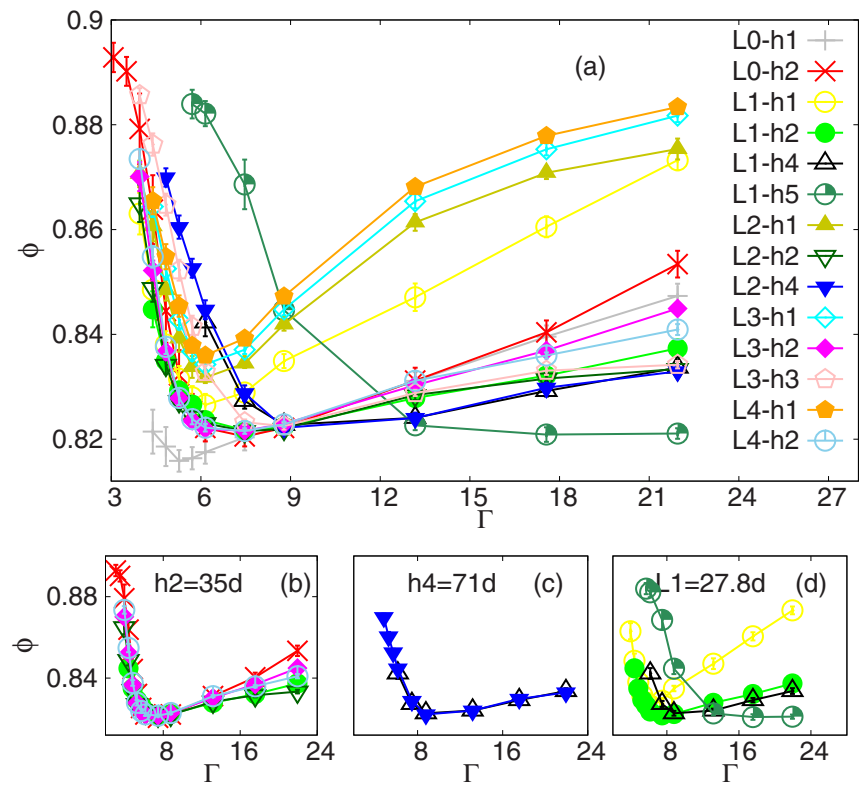

FIG. 3. (Color online) Packing fraction $\phi$ as a function of the reduced acceleration $\Gamma$. (a) All systems studied. [(b) and (c)] Only systems corresponding to $h 2$ and $h 4$, respectively. (d) Only systems corresponding to $L 1$. Error bars correspond to the standard deviation over the 20 independent realizations of the tapping protocol. 

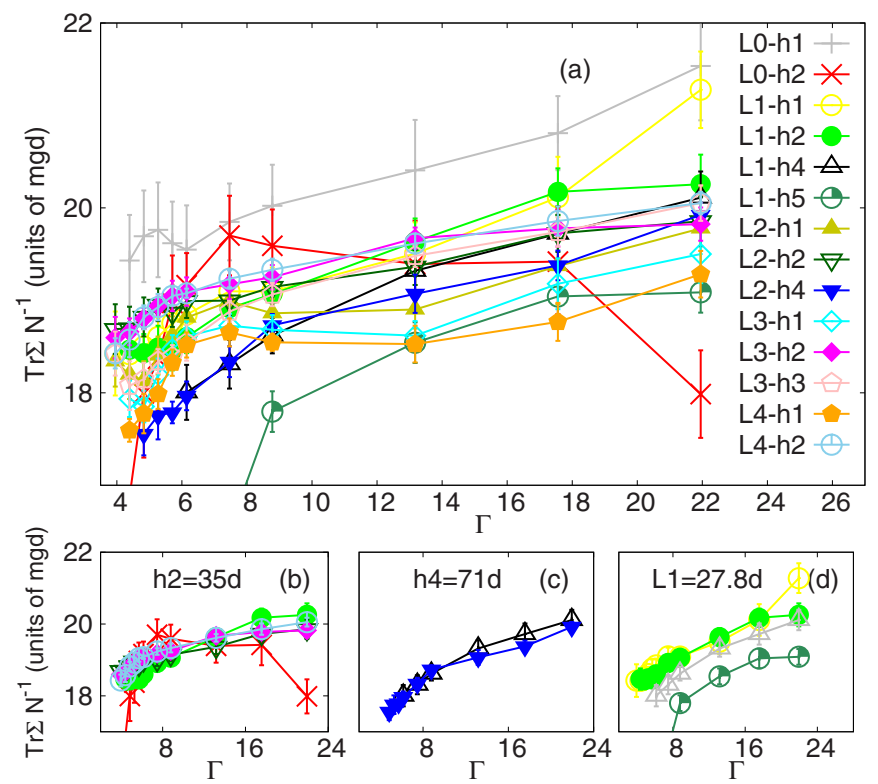

FIG. 4. (Color online) (a) Trace of $\Sigma$ as a function of $\Gamma$ for all samples studied. [(b) and (c)] Same as part (a) for the systems corresponding to $h 2$ and $h 4$, respectively. (d) Same as (a) including only systems corresponding to $L 1$. Error bars correspond to the standard deviation over the 20 independent realizations.

to a shallow bed will induce a more significant motion of the grains than in a tall granular column. In particular, the layer of interest (at a prescribed depth) will be reached by the perturbation after traversing different amounts of granular material. In Sec. V we will focus on this phenomenon and will suggest the use of a parameter, in replacement of $\Gamma$, that accounts for the effective perturbation felt by the granular bed.

\section{B. Force moment tensor}

Figure 4(a) shows the trace $\operatorname{Tr} \Sigma$ of the force moment tensor (averaged over all particles in the layer of interest) as a function of $\Gamma$. We take $\operatorname{Tr} \Sigma=\Sigma_{x x}+\Sigma_{z z}$. The component perpendicular to the cell plane $\Sigma_{y y}$ is not considered because it is at least one order of magnitude smaller than the other diagonal components. All curves in Fig. 4(a) are monotonic as was reported previously for two-dimensional systems [7,8]. We have seen that $\Sigma-\Gamma$ is actually nonmonotonic if smaller $\Gamma$ are consider [22]. However, $\Sigma$ is not well defined for low $\Gamma$ since independent realizations yield different (distinguishable) stationary states compatible with ergodicity breaking [22,23]. In all results reported here we have avoided low tap intensities where such phenomenon prevents a reproducible measure of $\Sigma$ and $\phi$.

Figures 4(b) and 4(c) show $\operatorname{Tr} \Sigma$ versus $\Gamma$ for two different system heights ( $h 2$ and $h 4$, respectively) and various cell widths. Figure 4(d) shows the results only for systems corresponding to $L 1$ and different nominal heights. In these figures we can see a similar behavior to the one observed for $\phi$ versus $\Gamma$ (see Fig. 3). Except for narrow systems [see, for example, the red crosses curve corresponding to $L=L 0$ and possibly the green solid circles curve for $L=L 1$ in Fig. 4(b)], columns with the same height show similar results, and curves corresponding to systems with different heights appear shifted. Since results for different $L$ with a given $h$ collapse, comparison of systems with the same aspect ratio $(h / L)$ yield the same shift as observed in Fig. 4(d).

As observed in the behavior of the steady-state packing fraction, the steady-state force moment tensor is also well reproduced for a given tap intensity and $h$ if $L$ is not too small. Typically, $L>L 1$ warrants a reasonable collapse. However, the height of the system is an important parameter that influences the stress state of the layer of interest. Once again, this is due to the different effective perturbation acting on the sample since the pulse has to propagate through columns of different heights. Hence, a change in the nominal height of the sample will effectively induce a layer (located at the same depth in both samples) to visit different steady states. In Sec. IV D this will be more clear when we plot the loci of the steady states visited in the $\phi-\Sigma$ phase diagram.

\section{Fluctuations}

Previous works [27] have shown that in granular systems the presence of correlations imply that density fluctuations $\Delta \phi$ do not scale with $\sqrt{N_{a}}$, with $N_{a}$ being the number of particles in the region of analysis. Nevertheless, we have analyzed regions of different sizes and have observed that it is possible to scale $\Delta \phi$ with $\sqrt{N_{a}}$ if we use layers sufficiently thick [28]. In particular, we find that for layers thicker than $15 d, \Delta \phi \sqrt{N_{a}}$ and $\Delta \Sigma \sqrt{N_{a}}$ converge to well-defined values. Hence, we have used layers of this thickness to carry out the analysis of the fluctuations. Notice that such regions in our systems with $L>L 1$ contain at least twice as many grains as the larger regions considered in Ref. [27].

In Fig. 5(a) we plot $\Delta \phi$ as a function of $\phi$ for a set of characteristic system sizes. We can see that different curves are shifted in this plot. This is in part because the regions of analysis comprise different numbers of grains (they have different widths) but also in part because systems of different heights are compared, which visit different steady states, as we mentioned in the previous section.

In Figs. 5(b) and 5(c) we scale the fluctuations with the expected system size dependency and separate results from different heights. The curves of $\Delta \phi \sqrt{N_{a}}$ as a function of $\phi$ for a given $h$ collapse rather well for the various $L$. We have not included here results for narrow columns for which curves do not collapse. For $h 4$ this means that only systems with $L \geqslant L 1$ must be considered; however, for $h 2$ columns as wide as $L 2$ are necessary. In general, choosing $L \geqslant L 2$ seems to warrant a reasonable independence of the fluctuations with the system width. If we compare the scaled fluctuations for two different $h$ [see Fig. 5(d)], we observe that $\Delta \phi \sqrt{N_{a}}$ differ for a given $\phi$ at high packing fractions (corresponding to the lower tap intensities). If two steady states present the same $\phi$ but different fluctuations one expects that the states are distinguishable. Indeed, in this case the force moment tensors of these states differ [see corresponding left branch of the curves in Fig. 5(h), where $\operatorname{Tr} \Sigma$ is shown]. Interestingly, for low $\phi$, fluctuations coincide even for these samples of different $h$. However, this does not warrant that the states are the same. In fact, we have seen that $\Sigma$ for these states is similar but distinguishable.

In Figs. 5(e), 5(f), 5(g), and 5(d) we can see similar results for the force moment tensor. In this case it becomes much clearer that the fluctuations of $\Sigma$ for systems with the same 

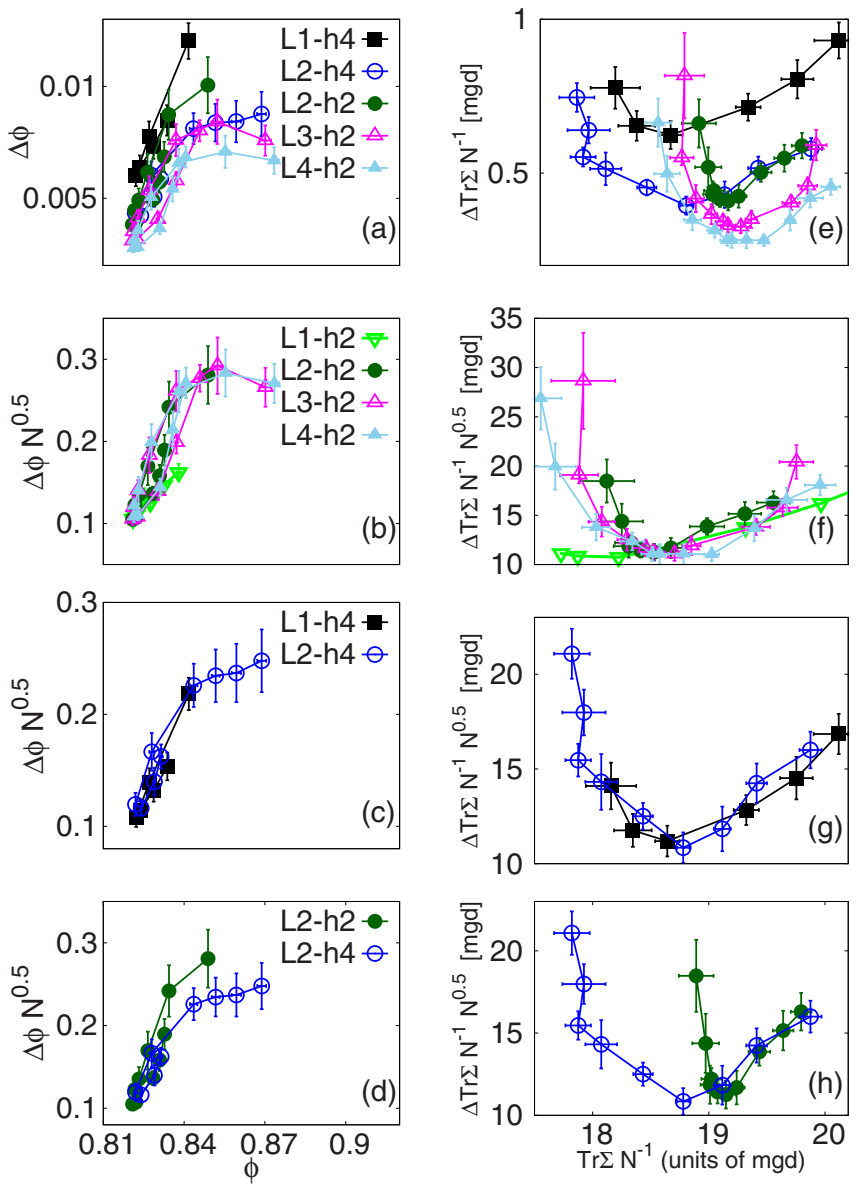

FIG. 5. (Color online) (a) $\Delta \phi$ as a function of $\phi$. [(b), (c), and (d)] $\Delta \phi \sqrt{N_{a}}$ as a function of $\phi$ for systems corresponding to $h 2$, $h 4$, and $L 2$, respectively. (e) $\Delta \operatorname{Tr} \Sigma$ versus $\operatorname{Tr} \Sigma$ for the same set of system sizes plotted in (a). [(f), (g), and (h)] $\Delta \operatorname{Tr} \Sigma \sqrt{N_{a}}$ for systems corresponding to $h 2, h 4$, and $L 2$, respectively. The layer of particles analyzed here is located at a depth of $10 d$ and is $15 d$ thick. Error bars correspond to the standard deviation over the 20 independent realizations.

height collapse with the $\sqrt{N_{a}}$ scaling, whereas fluctuations of systems with different $h$ do not.

\section{Phase diagram}

The packing fraction as a function of the force moment tensor seems to converge for systems with the same height (see Fig. 6) if they are wide enough (results for narrow systems, where this function does not collapse are not included in this plot). From Fig. 6, it becomes clear that systems with different heights visit different macroscopic stationary states when the same tapping protocol is used. Even if the same $\phi$ can be attained, the corresponding $\Sigma$ will differ (see the left region of the curves in Fig. 6 that correspond to the low tap intensities). As discussed in Ref. [7], the same $\phi$ may correspond to different $\Sigma$ values, for a given system size, given the nonmonotonic response of the packing fraction.

In Fig. 7(a) we can see $\phi$ as a function of $\operatorname{Tr} \Sigma$ for three different system heights. Again, it is possible to distinguish that systems corresponding to different $h$ visit different stationary equilibrium states. In Fig. 7(b) we plot $\phi$ as a function of

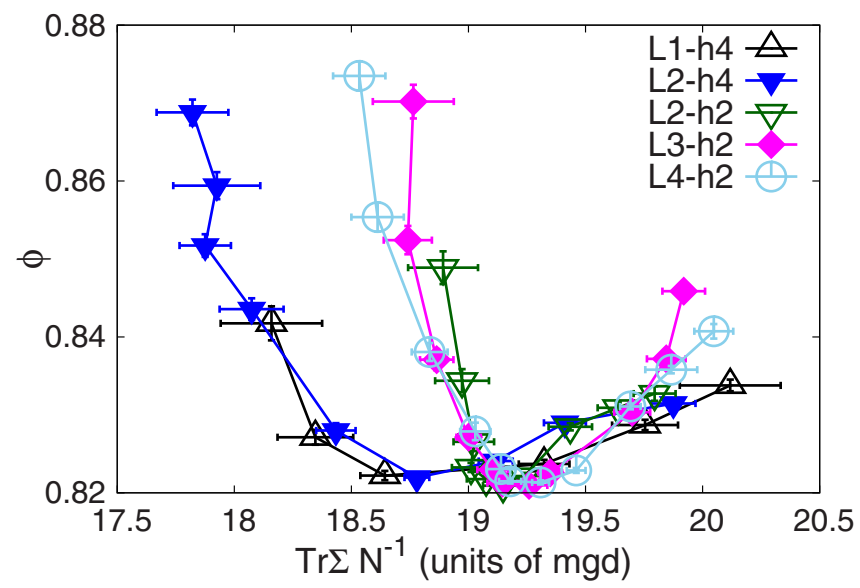

FIG. 6. (Color online) $\operatorname{Tr} \Sigma$ as a function of $\phi$ for two sets of systems with same height $h 2$ and $h 4$ and different widths. The layer of particles analyzed is located at a depth of $10 d$ and is $15 d$ thick.

$\Delta \phi \sqrt{N_{a}}$ for the same systems as in Fig. 7(a). Finally in Fig. 7(c) we can see $\Delta \operatorname{Tr} \Sigma \sqrt{N_{a}}$ as a function of $\operatorname{Tr} \Sigma$. Here we can observe that it is possible to tell apart fluctuations of $\phi$ for states with the same $\phi$ if they have different $\Sigma$. Conversely, it is also possible to distinguish fluctuations of $\Sigma$ for states with the same $\Sigma$ if they correspond to different $\phi$. We can also see that there exist some states with same $\phi$ and $\Sigma$ for systems with different heights. In such cases (see right branch that corresponds to high taps) the steady states exhibit similar fluctuations. This reinforces the hypothesis that $\phi$ and $\Sigma$ define the macroscopic stationary state fully. By changing the height of a granular column, a layer at a given depth will visit different regions of the phase diagram in a way similar to the effect obtained by changing the duration of the excitation pulse $[7,8]$. The effect of a thicker bed underneath the layer of
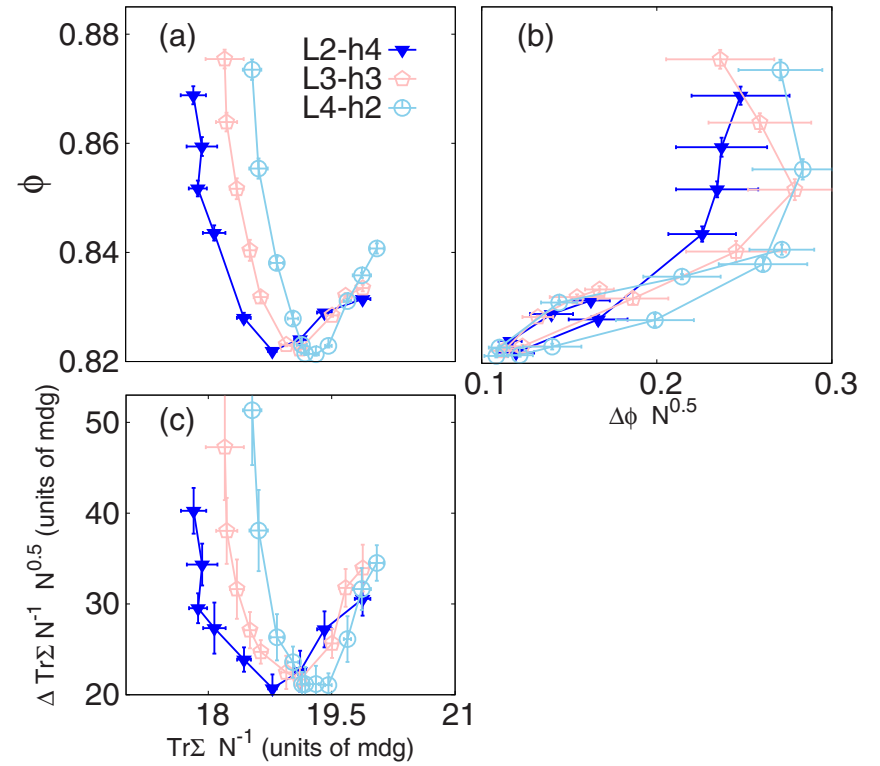

FIG. 7. (Color online) (a) $\phi$ as a function of $\operatorname{Tr} \Sigma$. (b) $\phi$ as a function of $\Delta \phi \sqrt{N_{a}}$. (c) $\Delta \operatorname{Tr} \Sigma \sqrt{N_{a}}$ as a function of $\operatorname{Tr} \Sigma$. The systems correspond to three different heights (and different widths). The layer of particles analyzed is located at a depth of $10 d$ and is $15 d$ thick. 
interest is to change the effective perturbation felt by the layer, leading to new states in the $\phi-\operatorname{Tr} \Sigma$ diagram.

\section{EFFECTIVE EXCITATION}

It is clear from Figs. 3-7 that the system height is a necessary parameter to predict the final macroscopic stationary state of a tapped granular sample. Samples of different width show consistent results if $L \gtrsim 50$ for $\phi$ and $\Sigma$ as well as for their fluctuations. However, if two systems have different $h$, even when layers at the same depth are considered, the values of $\phi$ and $\Sigma$ obtained for a given $\Gamma$ differ significantly. This is to be expected, as we mentioned, since the effective excitation felt by the layer will depend on the transmitted energy through the granular bed underneath. Moreover, even if steady states presenting the same $\phi$ are compared (whatever the necessary $\Gamma$ ) for two different $h$, the values of $\Sigma$ may not coincide (Fig. 6), indicating that the steady states do in fact differ. Hence, to be able to compare results obtained on different systems (e.g., from different laboratories) it is necessary to compare columns of same heights.

There has been some attention focused on finding a parameter alternative to $\Gamma$ to characterize the tap applied to the system. In particular, if different $\omega$ are used for the pulse, the corresponding $\phi-\Gamma$ curves appear shifted. It is expected that a suitable scaling may allow us to collapse all results into a single master curve for all $\omega$. In this way, a macrostate of given $\phi$ can be associated to a particular "effective" excitation done on the system. Dijksman and van Hecke investigated an annealing protocol similar to the one used in the present work (Sec. III A) but varying both amplitude and fundamental frequency of the pulse used to tap the system [13]. They proposed the use $\varepsilon=\Gamma T$ as the characteristic parameter of the tap, where $T$ is the period of the wave shape of the pulse. The authors concluded that this parameter results appropriate to collapse their different $\phi-\varepsilon$ curves. In a different work, Ludewig et al., investigating a similar problem, proposed to use a tap parameter related with the energy given to the system during the tap [5]. They took into account the potential and kinetic energy gained by the granular system in the time at which it looses contact with the cell floor, i.e. when its acceleration reaches the gravity acceleration. This parameter also allows to collapse curves obtained for different $\omega$. It is simple to show that, disregarding the marginal contribution of the potential energy included in the Ludewig's parameter, both parameters (Dijksman's and Ludewig's) are equivalent in essence. In both cases the effective parameter depends on $\Gamma T \equiv A \omega$. In Ref. [13] this dependence is linear while in Ref. [5] it is quadratic. More importantly, the former parameters try to unify the results just by introducing scale factors that depend only on the external input applied to the systems ignoring the geometrical details of the container.

In the present work we have a set of curves obtained with same $\omega$, but they look shifted if they correspond to systems with different heights [see Fig. 3(d)]. The effective parameters of Refs. [13] and [5] are not useful in this case because they do not take into account the system height. Pugnaloni et al. proposed to use the "effective expansion," $\varepsilon$, of the granular pack during the system flight to characterize the effect of the tap on the system [6]. This parameter allows for the comparison
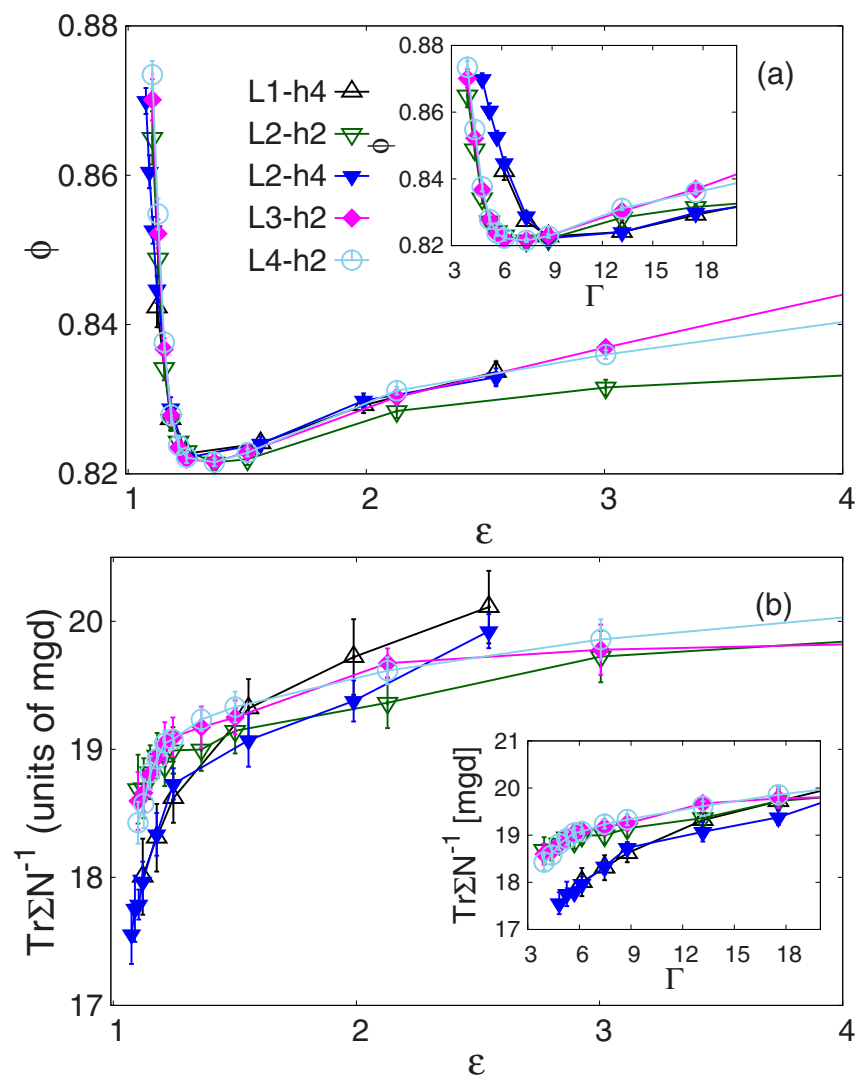

FIG. 8. (Color online) $\phi$ (a) and $\operatorname{Tr} \Sigma$ (b) as a function of $\varepsilon$ [see Eq. (2)], corresponding to systems with $L 1$ and different heights. The insets show the same data plotted as a function of $\Gamma$.

of results corresponding to different numerical models. Here we will follow this approach by defining $\varepsilon$ as the "effective flight" of the sample in the sense that the actual height reached by the grains during the tap is compared with the typical height of the sample at rest. Hence,

$$
\varepsilon=\frac{h_{\max }}{h_{0}},
$$

where $h_{\max }$ is the maximum height of the center of mass of the sample during the flight and $h_{0}$ is the typical height at rest. This can be thought of as a crude indication of how much free space is typically introduced in the pack during a tap. ${ }^{1}$

Taking into account the sine shape of the perturbation applied and neglecting dissipation against the walls or between the particles, we can estimate $h_{\max }=A^{2} \omega^{2} /(2 g)+h_{0}$. Here, $A^{2} \omega^{2} /(2 g)$ corresponds to the flight distance approximating the lift off velocity by $A \omega .^{2}$ Hence,

$$
\varepsilon=\frac{A^{2} \omega^{2}}{(2 g) h_{0}}+1 .
$$

Figure 8(a) shows $\phi$ as a function of $\varepsilon$ for systems with different heights. We can see that results are similar now,

\footnotetext{
${ }^{1}$ The expansion of a granular sample during each tap is in fact rather inhomogeneous in the vertical direction.

${ }^{2}$ This is not the actual liftoff velocity but the maximum velocity of the cell which is actually an overestimate.
} 
particularly at low tap intensities [cf. inset of Fig. 8(a) where the same data is shown as a function of $\Gamma]$. This suggests that macroscopic steady states from granular columns with different $h$ may be the same state if they are tapped with the same $\varepsilon$ rather than the same $\Gamma$. Unfortunately, this is not the case as we show in the following paragraph.

Figure 8(b) presents $\operatorname{Tr} \Sigma$ as a function of $\varepsilon$ for the same systems as in Fig. 8(a). Here curves look more similar in comparison with the representation in terms of $\Gamma$ (see inset). However, for low $\varepsilon, \operatorname{Tr} \Sigma$ is distinguishably smaller for taller systems. Hence, even if for a given $\varepsilon$ the steady states of columns with different $h$ seem to be the same in terms of packing fraction, they present different stress state. We believe this poorer collapse in $\Sigma$ is related to the fact that even if we can estimate the effective flight of a layer of material at the same depth for systems of different high, the actual stress state after deposition will depend also on the effective mechanical properties of the bed underneath. Taller columns provide a thicker bed below the layer of interest. This enables the layer under consideration to dissipate energy more efficiently by "landing on a softer substrate." This would lead to the lower values of $\Sigma$ observed. A tentative explanation of this contrast on the behavior of $\phi$ and $\Sigma$ could be related with the interplay existing between geometrical and structural degrees of freedom as has been recently discussed by Blumenfeld et al. [29]. Indeed, the average volume fraction depends in a nontrivial way not only on the coordination number but also on the global stress imposed on the boundaries of the system. Hence, although small effective flights induce comparable volume fractions, the stress at the boundaries strongly depends on the small particle rearrangements observed at this tap amplitudes as is obvious from Fig. 8.

It is worth emphasizing that $\varepsilon$ also results a function of $A \omega$ as in Refs. [5,13]. However, in addition, Eq. (2) takes into account the system height $h$. Then $\varepsilon$ could be used to compare results on $\phi$ for systems of different heights, bearing in mind that packing fraction on its own cannot fully describe the state and hence $\Sigma$ may not be the same.

\section{CONCLUSIONS}

We have considered the steady state of tapped granular columns for different system sizes by measuring the packing fraction, the force moment tensor, and their fluctuations. We have shown that for systems of the same height, $\phi, \Sigma$ and their fluctuations converge to well-defined values as the column width grows beyond $\approx 50 \mathrm{~d}$. This is observed if a thin layer of grains at a fixed depth is considered in the analysis with the provision that the layer follows the profile of the free surface.
The thickness of the layer of interest becomes irrelevant above $\approx 3 d$ except if fluctuations are measured. For the calculation of fluctuations, layers at least $15 d$ thick are necessary to find consistent results scaling with $\sqrt{N}$.

An important number of experiments and simulations are carried out on relatively small systems. Our results indicate that in many cases these system sizes do not meet the minimum required to warrant that results are insensitive to the particular geometrical dimensions chosen. In spite of the fact that we restrict our analysis to $2 \mathrm{D}$ systems, the same arguments seem to be valid for the $3 \mathrm{D}$ case.

We have found that the nonmonotonic behavior of the packing fraction as a function of the tap intensity [6-8] is still present if we change the systems size. However, the minimum $\phi$ is reached at higher tap intensities for taller systems. Likewise, we have observed that the fluctuations of the packing fraction as a function of $\phi$ exhibit a concave down behavior in agreement with previous studies [7,8].

For systems of different height, the values of $\phi$ and $\Sigma$ obtained for a given tap amplitude differ. We proposed the use of the effective flight $\varepsilon$ to characterize the effect of the tap on the granular layer of interest; speculating that a suitable scaling would allow the collapse of data for different $h$. We showed that as a function of $\varepsilon$ the different $\phi$ curves achieve a reasonable collapse. However, the $\Sigma-\varepsilon$ curves do not collapse in the full range of $\varepsilon$. Taller columns show consistently smaller values of $\Sigma$ than shallow columns at low $\varepsilon$. We have shown that systems of different heights lead the layer under study to visit different loci in the $\phi-\Sigma$ phase space. Hence, the effective perturbation felt by the layer of interest is affected by the column of grains underneath leading to new steady states. We argue that there are two main ingredients in the effect of the underlying column of grains. On the one hand, the effective expansion of the layer will be shifted by a factor $h$ [see Eq. (2)]. On the other hand, the layer will land on a bed with different effective mechanical properties.

In summary, to be able to reproduce a steady state of tapped granulars, not only the amplitude $A$ and shape (e.g., $\omega$ ) of the pulse has to be considered; also the height of the column is essential, while the width can be disregarded as long as $L \gtrsim 50 d$ in 2 D.

\section{ACKNOWLEDGMENTS}

This work was supported by CONICET (Argentina), ANPCyT (Grant PICT 2012-2155, Argentina), and MINECO (Grant FIS2011-26675, Spain). D. Maza thanks Ministerio de Ciencia Tecnología e Innovación Productiva (Grant RAICES, Argentina).
[1] S. F. Edwards and R. B. S. Oakeshott, Theory of powders, Physica A 157, 1080 (1989).

[2] E. R. Nowak, J. B. Knight, M. L. Povinelli, H. M. Jaeger, and S. R. Nagel, Reversibility and irreversibility in the packing of vibrated granular material, Powder Tech. 94, 79 (1997).
[3] E. R. Nowak, J. B. Knight, E. Ben-Naim, H. M. Jaeger, and S. R. Nagel, Density fluctuations in vibrated granular materials, Phys. Rev. E 57, 1971 (1998).

[4] Ph. Ribière, P. Richard, P. Philippe, D. Bideau, and R. Delannay, On the existence of stationary states during granular compaction, Eur. Phys. J. E 22, 249 (2007). 
[5] F. Ludewig, S. Dorbolo, T. Gilet, and N. Vandewalle, Energetic approach for the characterization of taps in granular compaction, Europhys. Lett. 84, 44001 (2008).

[6] L. A. Pugnaloni, M. Mizrahi, C. M. Carlevaro, and F. Vericat, Nonmonotonic reversible branch in four model granular beds subjected to vertical vibration, Phys. Rev. E 78, 051305 (2008).

[7] L. A. Pugnaloni, I. Sánchez, P. A. Gago, J. Damas, I. Zuriguel, and D. Maza, Towards a relevant set of state variables to describe static granular packings, Phys. Rev. E 82, 050301 (2010).

[8] L. A. Pugnaloni, J. Damas, I. Zuriguel, and D. Maza, Master curves for the stress tensor invariants in stationary states of static granular beds. implications for the thermodynamic phase space, Papers Phys. 3, 030004 (2011).

[9] P. Richard, M. Nicodemi, R. Delannay, P. Ribiere, and D. Bideau, Slow relaxation and compaction of granular systems, Nat. Mater. 4, 121 (2005).

[10] M. P. Ciamarra, A. Coniglio, and M. Nicodemi, Thermodynamics and statistical mechanics of dense granular media, Phys. Rev. Lett. 97, 158001 (2006).

[11] M. Schröter, D. I. Goldman, and H. L. Swinney, Stationary state volume fluctuations in a granular medium, Phys. Rev. E 71, 030301 (2005).

[12] M. Nicodemi, A. Coniglio, and H. J. Herrmann, Frustration and slow dynamics of granular packings, Phys. Rev. E 55, 3962 (1997).

[13] J. A. Dijksman and M. van Hecke, The role of tap duration for the steady-state density of vibrated granular media, Europhys. Lett. 88, 44001 (2009).

[14] R. Blumenfeld and S. F. Edwards, On granular stress statistics: Compactivity, angoricity, and some open issues, J. Phys. Chem. B 113, 3981 (2009).

[15] S. F. Edwards, The full canonical ensemble of a granular system, Physica A 353, 114 (2005).

[16] S. Henkes, C. S. OHern, and B. Chakraborty, Entropy and temperature of a static granular assembly: An ab initio approach, Phys. Rev. Lett. 99, 038002 (2007).
[17] S. Plimpton, P. Crozier, and A. Thompson, LAMMPS-LargeScale Atomic/Molecular Massively Parallel Simulator (Sandia National Laboratories, 2007).

[18] S. Ardanza-Trevijano, I. Zuriguel, R. Arévalo, and D. Maza, Topological analysis of tapped granular media using persistent homology, Phys. Rev. E 89, 052212 (2014).

[19] N. V. Brilliantov, F. Spahn, J. M. Hertzsch, and T. Pöschel, Model for collisions in granular gases, Phys. Rev. E 53, 5382 (1996).

[20] L. E. Silbert, D. Ertaş, G. S. Grest, T. C. Halsey, D. Levine, and S. J. Plimpton, Granular flow down an inclined plane: Bagnold scaling and rheology, Phys. Rev. E 64, 051302 (2001).

[21] J. B. Knight, C. G. Fandrich, C. N. Lau, H. M. Jaeger, and S. R. Nagel, Density relaxation in a vibrated granular material, Phys. Rev. E 51, 3957 (1995).

[22] P. A. Gago, L. A. Pugnaloni, and D. Maza (unpublished).

[23] F. Paillusson and D. Frenkel, Probing ergodicity in granular matter, Phys. Rev. Lett. 109, 208001 (2012).

[24] P. Evesque and J. Rajchenbach, Instability in a sand heap, Phys. Rev. Lett. 62, 44 (1989).

[25] J. E. Macías, M. G. Clerc, C. Falcón, and M. A. García-Ñustes, Spatially modulated kinks in shallow granular layers, Phys. Rev. E 88, 020201 (2013).

[26] P. A. Gago, N. E. Bueno, and L. A. Pugnaloni, High intensity tapping regime in a frustrated lattice gas model of granular compaction, Granul. Matter 11, 365 (2009).

[27] F. Lechenault, F. da Cruz, O. Dauchot, and E. Bertin, Free volume distributions and compactivity measurement in a bidimensional granular packing, J. Stat. Mech. (2006) P07009.

[28] P. A. Gago, Variables de estado en la descripción estadística de empaquetamientos granulares estáticos. dependencia con el tamaño del sistema, Ph.D. thesis, Universidad Nacional de La Plata (2014).

[29] R. Blumenfeld and S. F. Edwards, Granular statistical mechanics-A personal perspective, Eur. Phys. J. Spec. Top. 223, 2189 (2014). 